\title{
Further Insight into the Brain's Rich-Club Architecture
}

\author{
Mangor Pedersen ${ }^{1,2}$ and Amir Omidvarnia ${ }^{1,2}$ \\ ${ }^{1}$ The Florey Institute of Neuroscience and Mental Health and ${ }^{2}$ Florey Department of Neuroscience and Mental Health, The University of Melbourne, Austin \\ Campus, Heidelberg, Victoria 3084, Australia \\ Review of Nigam et al.
}

The pioneering field of connectomics has the ambitious aim of mapping and understanding the connections of the brain. Brain connectivity can be analyzed at several spatial resolutions: macroscopic (whole-brain), mesoscopic (neuronal populations), and microscopic (single-neuron) scales (Bullmore and Sporns, 2009; Van Essen and Ugurbil, 2012). An important discovery in connectomics at the macroscale is the existence of a rich-club in the brain (van den Heuvel and Sporns, 2011). Members of this club constitute a few "rich" brain regions responsible for distributing a large fraction of the brain's neural communications. Using diffusion magnetic resonance imaging, the rich-club has been shown to include brain areas such as the precuneus, superior frontal/parietal lobules, hippocampus, and thalamostriatal structures in human subjects (van den Heuvel and Sporns, 2011). The implication is a high percentage of intercortical connections travel through these central hubs of information flow. So far, the rich-club has been conceptualized as a largescale brain network phenomenon in adult humans (van den Heuvel and Sporns,

Received March 8, 2016; revised April 7, 2016; accepted April 11, 2016.

We thank Graeme Jackson and Evan Curwood for insightful discussions and revisions. The Florey Institute of Neuroscience and Mental Health acknowledges the strong support from the Victorian Government and in particular the funding from the 0 perational Infrastructure. M.P is supported by The University of Melbourne scholarships (MIRS \& MIFRS).

The authors declare no competing financial interests.

Correspondence should be addressed to Mangor Pedersen, Florey Institute of Neuroscience and Mental Health, Melbourne Brain Centre, 245 Burgundy Street, Heidelberg, Victoria 3084, Australia. Fax +6139035 7381. E-mail:m.pedersen@brain.org.au.

DOI:10.1523/JNEUROSCI.0754-16.2016

Copyright $\odot 2016$ the authors $\quad 0270-6474 / 16 / 365675-02 \$ 15.00 / 0$
2011), human newborns (Ball et al., 2014), macaque monkey (Harriger et al., 2012), the nematode worm Caenorhabditis elegans (Towlson et al., 2013), and mouse hippocampus (Schroeter et al., 2015).

In a recent study published in The Journal of Neuroscience, Nigam et al. (2016) provided new evidence regarding the brain's rich-club architecture. These authors demonstrate that the macroscale rich-club organization of the brain extends to the microscale organization of cortical networks formed by closely spaced neurons. In these neuronal networks, a low percentage $(\sim 20 \%)$ of the neurons are responsible for a high percentage $(\sim 70 \%)$ of the incoming and outgoing information flow.

To measure the directional information flow between neurons, Nigam et al. (2016) rigorously analyzed effective connectivity based on in vitro (organotypic cultures) and in vivo microelectrode recordings in awake behaving mice. Their multistep analysis was designed to minimize spurious neuronal transactions. Transfer entropy, a model-free measure of effective connectivity, was adapted for quantifying single neuronal interactions (Vicente et al., 2011). This allowed the nonlinear and directional relationships of neural firing to be recorded. Using the transfer entropy measure, effective connectivity between hundreds of individual neurons was estimated. This led to an asymmetric weight matrix for each recording. These matrix elements were controlled for three possible factors: (1) the neural firing rate, estimated as spike jitter- ing at the source neuron; (2) the network drive, which was defined as global effects over the whole network, such as global network synchronization during a burst; and (3) spurious delayed connections between two typical neurons with a similar driver.

Two separate brain graphs were extracted to quantify directed neural network information: a binary graph representing pairwise directional connections between neurons, and its associated weighted graph. Several network measures including information transfer, neuronal diversity, and rich-club coefficient were extracted from the weighted directed graphs. Each of these metrics accounts for a specific aspect of neuronal networks. For instance, the importance of a node or link in the overall dynamical network behavior is quantified by the dynamic importance measure, whereas incoming and outgoing information flow within neuronal interactions is characterized by the neuron diversity measure. All of these measures, however, contribute to detecting possible rich neural populations in cortical networks and understanding their properties.

Given that the rich-club was initially thought to be a macroscale phenomenon observed over a few selected brain regions, the current results obtained by Nigam et al. (2016) challenges our current view about rich brain regions and neurons. These "important" neurons are responsible for the majority of information flow in local neuronal networks. It represents a rich-club structure at the brain's neuronal level with a few nodes experiencing high volume of information traffic, remark- 
ably similar to what is observed in the largescale networks of the brain (van den Heuvel and Sporns, 2011). In fact, rich neurons in the local cortical networks act as central hubs handling efficient network-wide communications between the nodes. The study also highlights another important signature of rich-club neurons. These neurons have significantly higher firing rates and more diverse outgoing information than "non-rich" neurons. Indeed, the neural firing rate and information transfer values extracted from the pool of rich-club neurons represent lognormal distributions with only a few important nodes generating high rate of neural firing in local cortical networks.

A likely purpose of the brain's rich-club organization is to ensure efficient neuronal processing at lowest possible cost (van den Heuvel et al., 2012). Although rich brain nodes demand significant metabolic resources, their efficient information processing outweighs this high energy consumption (Towlson et al., 2013). This is a situation that can also be observed in other dynamically efficient systems such as international flight networks. Major airports in cities including London, Frankfurt, and Atlanta are highly costly to run and maintain. However, this cost will be toward their central role in efficiently connecting worldwide flight paths (Guimerà et al., 2005). In analogy, the brain's dynamical system can be conceived as a collection of interleaved costefficient networks where a few nodes (for example, brain regions and/or neurons) are responsible for a disproportionate amount of neural activity (Bullmore and Sporns, 2012). Towlson et al. (2013) suggested that the brain's rich-club stems from cost-minimization of information processing in scale-invariant neural activities. It is therefore fair to postulate that the brain's rich-club architecture evolved to meet exhaustive neural demands within the brain's allocated “energy budget” (Attwell and Laughlin, 2001).

The gap between the brain's rich-clubs at the macroscale (van den Heuvel and Sporns, 2011) and microscale (Nigam et al., 2016) may be filled by a recently published paper by Fulcher and Fornito (2016). In this study, Fulcher and Fornito (2016) analyzed rich-club properties of a mesoscale mouse connectome, derived from two-photon imaging of wholebrain connectivity at a near-neuronal level (Oh et al., 2014). The results showed a strong presence of rich-club arrangement in the mouse connectome. Additionally, rich-club nodes were strongly associated with genes responsible for metabolic regulation. This provides another piece of convincing evidence for the role of rich-clubs in governing costefficient networks.
The brain's rich-club organization at different spatial scales constitutes a potential ground for characterizing neural network deterioration resulting from disease. Preliminary studies suggest that the brain's rich-club may be selectively targeted in brain diseases including schizophrenia (van den Heuvel et al., 2013). This hypothesis is also supported by a comprehensive meta-analysis based on the data of more than 15,000 patients with various neurological or psychiatric conditions (Crossley et al., 2014). It shows that nearly all brain diseases are likely to be associated with some form of dysfunction in rich brain areas. These observations are in line with a functional magnetic resonance imaging simulation study showing that virtual resection of rich brain regions cause much more detrimental network effects compared with the damage of non-rich areas (Alstott et al., 2009). In summary, the potential role of the brain's rich-club at macro-, meso- and micro-scales in neural diseases deserves considerable attention.

From a technical point of view, it would be beneficial to compare and verify the current results by Nigam et al. (2016) using measures of directed neuronal connectivity comparable to that of transfer entropy. For example, measures based on Granger causality (Granger, 1969), a linear representation of directional information flow between signals, can provide a picture of causal interneural interactions that might be different from what the transfer entropy measure provides. As Nigam et al. (2016) pointed out, the "spurious link removal" procedure used here is relatively conservative in the sense that it may ignore partial and indirect relationships between neurons. Also, bivariate (pairwise) comparison between neurons may not provide an accurate picture of the brain neural networks that contains a lot of global interactions. Analysis of neuronal rich-club behavior using multivariate information transfer criteria offers a promising avenue for future research.

\section{References}

Alstott J, Breakspear M, Hagmann P, Cammoun L, Sporns O (2009) Modeling the impact of lesions in the human brain. PLoS Comput Biol 5:e1000408. CrossRef Medline

Attwell D, Laughlin SB (2001) An energy budget for signaling in the grey matter of the brain. J Cereb Blood Flow Metab 21:1133-1145. CrossRef Medline

Ball G, Aljabar P, Zebari S, Tusor N, Arichi T, Merchant N, Robinson EC, Ogundipe E, Rueckert D, Edwards AD, Counsell SJ (2014) Rich-club organization of the newborn human brain. Proc Natl Acad Sci U S A 111:7456-7461.

Bullmore E, Sporns O (2009) Complex brain networks: graph theoretical analysis of struc- tural and functional systems. Nat Rev Neurosci 10:186-198. CrossRef Medline

Bullmore E, Sporns O (2012) The economy of brain network organization. Nat Rev Neurosci 13:336-349. CrossRef Medline

Crossley NA, Mechelli A, Scott J, Carletti F, Fox PT, McGuire P, Bullmore ET (2014) The hubs of the human connectome are generally implicated in the anatomy of brain disorders. Brain 137:2382-2395. CrossRef Medline

Fulcher BD, Fornito A (2016) A transcriptional signature of hub connectivity in the mouse connectome. Proc Natl Acad Sci U S A 113: 1435-1440. CrossRef Medline

Granger CWJ (1969) Investigating causal relations by econometric models and crossspectral methods. Econometrica 37:424-438. CrossRef

Guimerà R, Mossa S, Turtschi A, Amaral LA (2005) The worldwide air transportation network: anomalous centrality, community structure, and cities' global roles. Proc Natl Acad Sci U S A 102:77947799. CrossRef Medline

Harriger L, van den Heuvel MP, Sporns O (2012) Rich club organization of macaque cerebral cortex and its role in network communication. PloS One 7:e46497. CrossRef Medline

Nigam S, Shimono M, Ito S, Yeh FC, Timme N, Myroshnychenko M, Lapish CC, Tosi Z, Hottowy P, Smith WC, Masmanidis SC, Litke AM, Sporns O, Beggs JM (2016) Rich-club organization in effective connectivity among cortical neurons. J Neurosci 36:670-684. CrossRef Medline

Oh SW, Harris JA, Ng L, Winslow B, Cain N, Mihalas S, Wang Q, Lau C, Kuan L, Henry AM, Mortrud MT, Ouellette B, Nguyen TN, Sorensen SA, Slaughterbeck CR, Wakeman W, Li Y, Feng D, Ho A, Nicholas E, et al. (2014) A mesoscale connectome of the mouse brain. Nature 508:207-214. CrossRef Medline

Schroeter MS, Charlesworth P, Kitzbichler MG, Paulsen O, Bullmore ET (2015) Emergence of rich-club topology and coordinated dynamics in development of hippocampal functional networks in vitro. J Neurosci 35: 5459-5470. CrossRef Medline

Towlson EK, Vértes PE, Ahnert SE, Schafer WR, Bullmore ET (2013) The rich club of the $C$. elegans neuronal connectome. J Neurosci 33: 6380-6387. CrossRef Medline

van den Heuvel MP, Sporns O (2011) Rich-club organization of the human connectome. J Neurosci 31:15775-15786. CrossRef Medline

van den Heuvel MP, Kahn RS, Goñi J, Sporns O (2012) High-cost, high-capacity backbone for global brain communication. Proc Natl Acad Sci U S A 109:11372-11377. CrossRef Medline

van den Heuvel MP, Sporns O, Collin G, Scheewe T, Mandl RC, Cahn W, Goñi J, Hulshoff Pol HE, Kahn RS (2013) Abnormal rich club organization and functional brain dynamics in schizophrenia. JAMA Psychiatry 70:783-792. CrossRef Medline

Van Essen DC, Ugurbil K (2012) The future of the human connectome. Neuroimage 62: 1299-1310. CrossRef Medline

Vicente R, Wibral M, Lindner M, Pipa G (2011) Transfer entropy: a model-free measure of effective connectivity for the neurosciences. J Comput Neurosci 30:45-67. CrossRef Medline 